\title{
Neuroscientists go wireless
}

\begin{abstract}
To study the neural complexities of animals moving though physical and social space, labs increasingly turn to wireless technology.
\end{abstract}

\section{Vivien Marx}

$\mathrm{B}$ ats dip and weave through the air to catch insects or stop to indulge at their favorite fruit tree; monkeys make strategic decisions as they forage in trees for food or cultivate social lives; archerfish catch lunch by shooting water at insects on branches hanging over the water; rats scurry across the ground or subway tracks, and also climb vertically and play hide and seek with humans. To understand such behavioral richness and assess the neural computation involved, scientists observe, videotape and measure ${ }^{1-4}$. The measurement readouts from the brain and nervous system might be electrophysiology data. Or teams might use optogenetic or electrical stimulation and assess how behavior changes. But they would like to do so in freely moving and behaving animals.

"With recording cables, you can't really have the rats go 'under' something and you certainly can't get them to climb through things," says Roddy Grieves, a postdoctoral fellow in Jeffrey Taube's lab at Dartmouth College. When birds have optical fibers sticking out of their heads, "flying, singing, is not going to happen that much anymore," says University of Arizona researcher Philipp Gutruf. "They only sing if they're super-comfortable." Labs want the animals to behave as closely to their natural behavior as possible. "I'm very much a believer in these more naturalistic developments," says Weizmann Institute neuroscientist Nachum Ulanovsky, who is writing a book on 'natural neuroscience'. Rodents like to chew cables, which can ruin a tethered experiment. If bats fly around with cabled systems, the result is a big knot. Especially in social neuroscience with experiments involving several animals, "wireless is a must," says Ulanovsky.

Wireless technology is rapidly advancing as an essential tool in neuroscience, and when designed right, these systems "can allow animals to behave much more normally then traditional tethered recordings," says Ronen Segev, a neuroscientist at Ben-Gurion University of the Negev in Israel. "In fish, this is especially true." Indeed, one can record electrophysiology data from the brains of swimming fish.

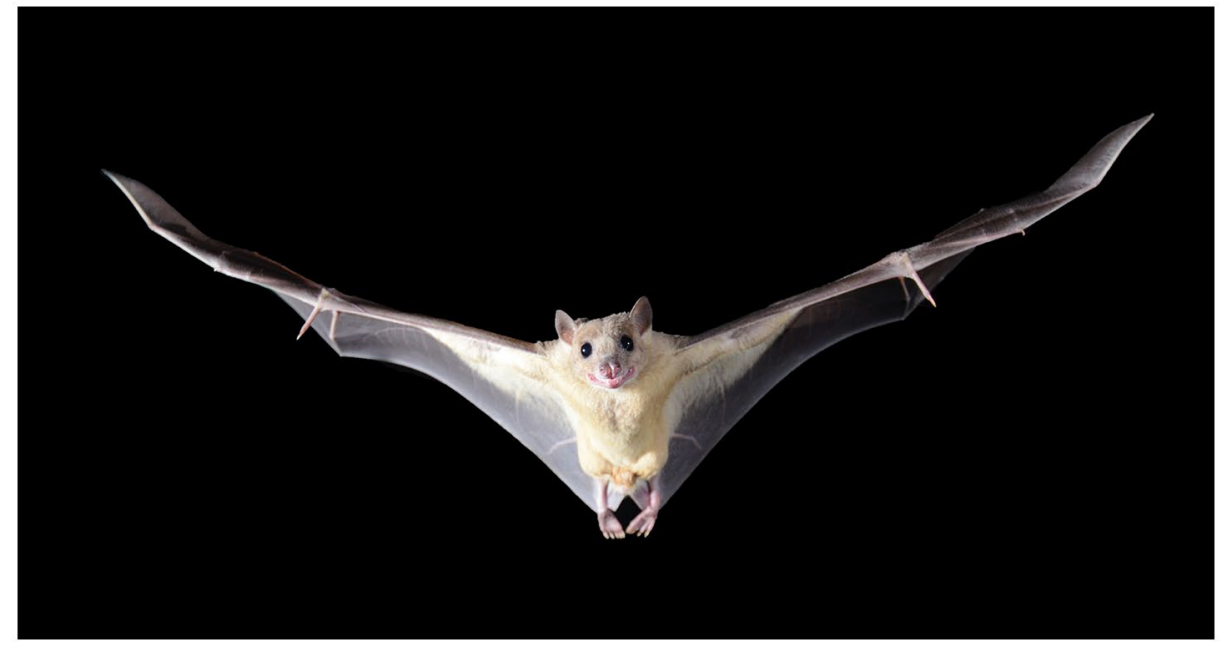

Bats are expert navigators. Their brains shed light on this expertise and on the mammalian brain more generally. Credit: I. Belson

To get the wireless systems they need, researchers team up with engineers to design, build and test. Some neuroscientists are themselves also physicists or engineers. Some of the resulting systems are battery driven while others deploy energy wirelessly. Sometimes data is transmitted wirelessly, other systems store data on board. Wireless systems in neuroscience are getting smaller and more robust, but troubleshooting is often needed given the high demands systems must address.

Naho Konoike, a neuroscientist at Kyoto University, uses wireless recording with marmosets to study sibling interaction, vocalization and the effects of hormones on social behavior. In a marmoset family, the team seeks to simultaneously record all movements, vocalizations and other behaviors, and then they comb through the collected 'big data'. Konoike is part of an international collaborative research effort that includes a device developer, zoologists, animal experimenters and a data analyst. "We believe that if the developers and experimenters become more close, we will be able to create better products for animal experiments," she says. Her requests for system developers include keeping in mind that labs need systems that minimize harm to living animals; that the systems should not be easily dislodged, which is especially challenging with monkeys; and that the systems need to be long-lasting, have a light battery and have a high ratio of signal to noise.

\section{D travel}

"Seeing these weird and wonderful mazes in papers was what attracted me to do my postdoc in Kate's lab," says Roddy Grieves about why he joined the lab of Kate Jeffery at University College London as a postdoctoral fellow. Her climbing walls for rats or spiral staircase enabled work beyond 2D experimental designs, using 3D mazes to explore how navigation is encoded in the brain. The team, including Grieves, built a see-through lattice maze around 1 cubic meter in volume that rats climb through to forage for malt paste, a snack favorite. "We stuck little patches of it to the maze for the rats to search for," says Grieves. With five cameras, the scientists videotaped the animals and triangulated their positions.

It's known that as a rat walks around in $2 \mathrm{D}$, spatial neurons in the hippocampus are active, as are grid cells in the entorhinal 


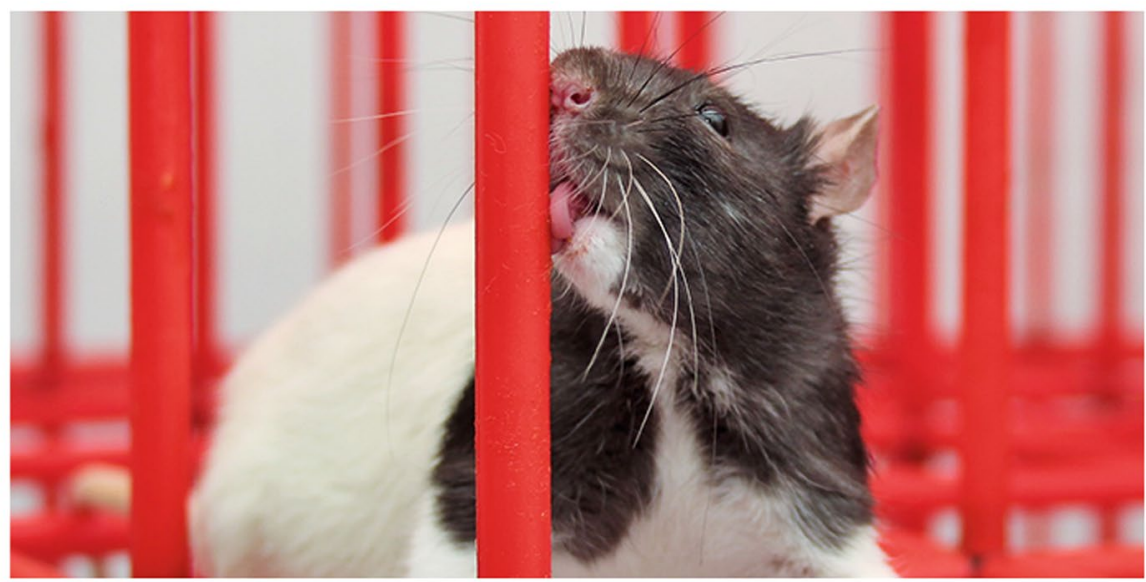

Kate Jeffery, Roddy Grieves and colleagues used a see-through maze to wirelessly characterize how grid cells in the rat brain represent 3D space as the rat navigates through it. Credit: R. Grieves

cortex, a brain region associated with the hippocampus ${ }^{5}$. Grid cells fire in a repetitive spatial pattern as an animal moves through a space. "What astounded everyone was that if you map out the 'patches' of firing they form a hexagonal pattern that covers the environment," says Grieves These 'firing fields' of the grid cells in the brain are a regular array, which lines up with theoretical work that dates back to the nineteenth century, he says. It has been an open question how the grid cell firing fields are arranged when an animal navigates a 3D environment. Computational models had shown mixed results, "so going into the experiment we weren't really sure what to expect," says Grieves.

In their $3 \mathrm{D}$ experiment ${ }^{6}$ they used a wireless system to record electrophysiological data. Tetrodes, which are clusters of four electrodes, and associated electronics were connected to a headstage on the animal, a 64-channel system from Triangle BioSystems, now Harvard Bioscience. Company scientists advised the team on their experimental setup. Signals were transmitted to a base station wirelessly via an antenna. The signal traveled ultimately to an Axona recording system. Along the way it was filtered and was amplified 100-fold. The wireless system replaced the traditional tether system the lab had previously used. "This worked really well for us because it meant we could utilize the decades of development that have gone into wired Axona recording systems but also have the convenience of wireless recording," he says.

The results surprised the team: when rats explore the $3 \mathrm{D}$ space of the lattice, grid-cell firing fields were not regularly stacked spherical fields. The fields were irregularly distributed.
This finding from the Jeffery lab matches results published at the same time from the Ulanovsky lab at Weizmann and colleagues at Hebrew University. That team used a different kind of wireless system and the Egyptian fruit bat. As the animals flew around a space about $6 \times 4.5 \times 3$ meters in size, they videotaped the bats with a Neuralynx system and tracked the activity of the spatial neurons in the animals' medial entorhinal cortex, including grid cells, using a system from Deuteron Technologies the researchers helped to design.

The bats flew around and stopped occasionally at fruit nibble stations mounted at different heights in the darkened space. The study focused on $3 \mathrm{D}$ space representation in bats also as "a window for more general questions," says Ulanovsky. It's a way to better understand grid cells and how the mammalian brain represents large $3 \mathrm{D}$ spaces. In their experiment, the scientists found grid-cell firing fields to not have the expected "perfect periodicity," says Ulanovsky. The spherical firing fields were spaced more or less equidistant from one another but lacked an overarching structure. They had, he says, expected the spherical firing field spheres to be packed like oranges in a grocery store. "The result is certainly surprising," he says.

He has long been discussing grid cells with Nobel laureate Edvard Moser, a researcher at the Norwegian University of Science and Technology, who has told him he is intrigued by this new result. In hindsight, says Ulanovsky, when they began this study, he and his team had preconceptions, almost a "research bias," he says. It took them a while to realize "we actually found something very different."

\section{Designed together}

For a decade, Ulanovsky has been using wireless systems and has worked with several companies. To develop the neural logger system they use with bats, seven years ago he began working with engineer Jacob Vecht and team at Deuteron Technologies. Ulanovsky likes the intense back and forth needed to address complex neuroscience questions and build custom wireless systems. Among the many technical aspects are signal-to-noise ratios, a need for stable recording data and generally much iteration and testing. This interplay is "grounding on both sides," says Ulanovsky. The relationship will continue with his new project that involves bats flying in a new 700-meter tunnel under construction. This expands on the lab's previous work in which the bats flew in a 200-meter tunnel equipped with fruit nibbling stations ${ }^{8}$. Electrophysiology data from another type of spatial neuron, place cells, were recorded.

For the latest grid-cell-focused experiment, the team's system includes electronics to drive tetrodes that are surgically lowered into the bat's brain. After surgery and healing, the connector to this device sticks out of the bat's head. When the bats are readied to fly, the Deuteron device is attached to the connector. It weighs around seven grams, which includes electronics, battery, a microSD card and a protective casing. Especially with larger distances, says Ulanovsky, one cannot transmit data wirelessly. The data are stored on the animal for later download and processing. "Most of the weight of our system is actually the battery," he says. They are currently working with Deuteron's new 64-channel system. "The systems are dropping in weight dramatically," he says.

For now, says Grieves, the wireless neuroscience field is split between such neural loggers and the system type he and his colleagues used. When transmitting "very precious neural data" wirelessly, interference and other issues can get in the way whereas neural loggers hold the data for later download. With the University College London experiment, they decided to capitalize on their existing recording systems and expertise for processing spikes, says Grieves. Neural loggers are an approach he thinks "will win out in the end" and would be his system of choice were the experiment repeated. The device saves raw, unprocessed data, which means more post-processing work than with their other system.

The work on $3 \mathrm{D}$ navigation and wireless approaches invites next steps. With the results from both rats and bats, it's now known the perfect sphere packing arrangements are not how the brain maps 
space, says Grieves. "Now we need to understand why that is the case and what it means for grid cells more generally." To do so will also take technical progress. "If I had to pick something, I would say that we are mainly limited by battery weight and capacity at this point, though; this is the technology that needs some advancement now," he says.

Neural loggers are "a terrific use of the low weights achieved for data loggers over the past ten years," says Ian Halpern, and "the ratio of size to rich data acquisition is high here." Halpern has long developed neuroscience instruments and co-founded the company Modular Bionics.

\section{Custom-build}

Deuteron Technologies founder Jacob Vecht worked previously as a staff electronics engineer, then struck out on his own to develop custom systems. He learned of the electrophysiology community's woes with wireless systems as labs used analog radio signaling. "They were getting horrible signals full of random noise," says Vecht. Newer systems use Wi-Fi that have high power needs and data transmission can get interrupted. Among other developments, in his view, 'Open Ephys' initiatives from a number of labs have helped to bring about smaller, less expensive systems. Vecht likes getting involved with neuroscience labs such as Ulanovsky's who have a "tricky game to play" in their need of reliable, lightweight, affordable wireless systems. Recording from neurons means sampling 30,000 times per second. One Deuteron device has 64 channels and weighs 3 grams. It holds a 64-gigabyte memory card, and the battery can record electrophysiology data for two and a half hours. He also has one with 128 channels. Such systems move labs away from expensive equipment in a rack to less pricey systems placed on animals. The device faces wear and tear. The animal is "knocking it, it's peeing and pooping on it, it's bumping and thumping it," says Vecht. Or swimming with it. He is readying a new system for Ronen Segev, who captures data in freely swimming fish. "Certainly, working with fish has been a tremendous challenge to keep the electronics dry and the fish wet at all times," says Vecht.

Segev and his team study how archerfish take aim and shoot a jet of water at insect prey as it meanders on a branch as high as two meters above the waterline. The fish has a large optic tectum in its brain where visual and sensory information is processed. Because the fish cannot visually follow prey as, for example, a lion might, the archerfish shifts its gaze to a predicted future spot its prey will move to. It had been thought that such visual calculations take a cortex, which fish do not have. With a wireless system, Segev hopes to study archerfish behavior and visual processing. "At the

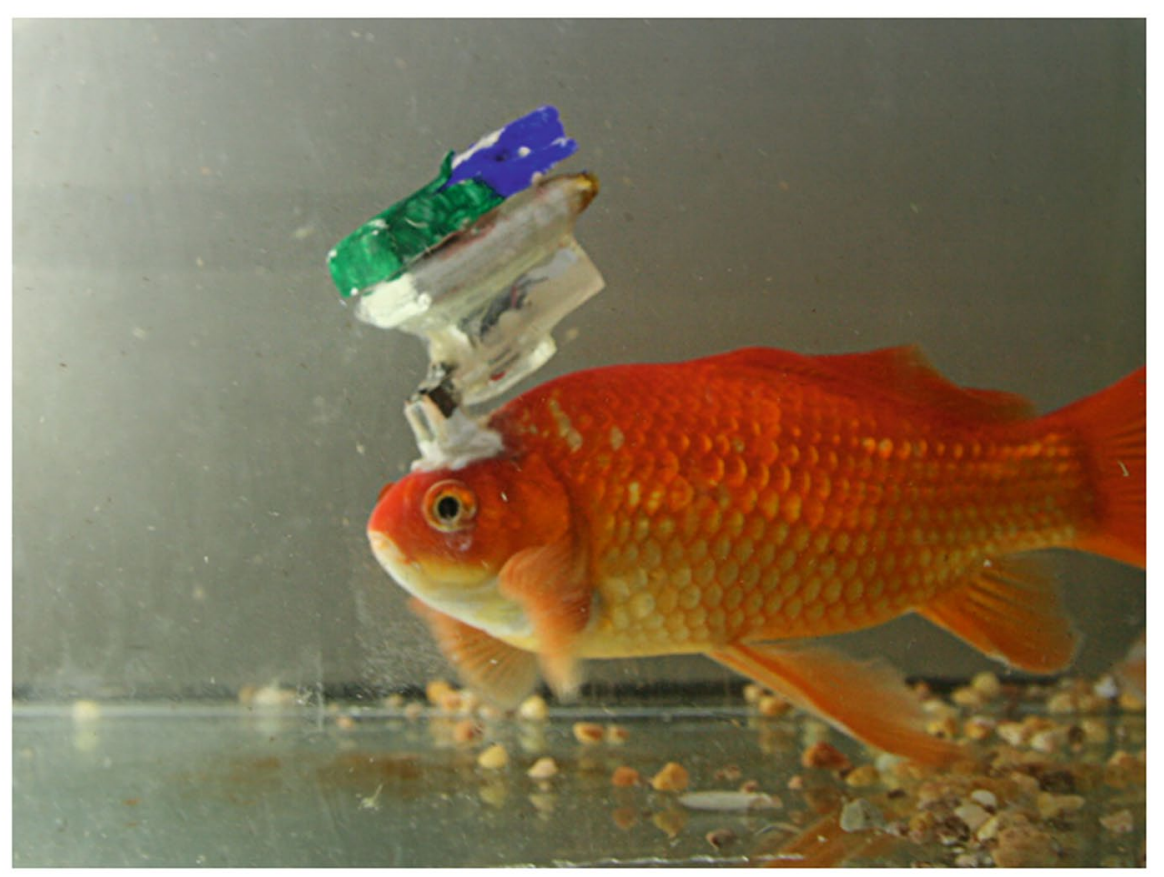

To better understand 3D navigation under water, it's possible to capture electrophysiology data from swimming fish. The colored Styrofoam block adds buoyancy. Credit: Segev lab, Ben-Gurion Univ. of the Negev

moment, the technology is not there yet," he says. The waterproof box they built for the electronics is too large for the archerfish to behave normally. For now they use a Deuteron neural logger to record from swimming goldfish to capture how the fish neurally encode 3D space. Goldfish have a brain region - the lateral pallium - that is homologous to the hippocampus, which handles navigation in the mammalian brain. When Segev and his team recorded from swimming fish, they found that cells in the goldfish's lateral pallium help the animal navigate through $3 \mathrm{D}$ space.

The scientists found border cells, head direction cells, and speed and velocity cells. Along with the neural logger, they add a low-tech flotation device, a piece of Styrofoam, for buoyancy. Even though it adds drag, the recording setup has made fish and other aquatic organisms "an opportunity for neuroscience," he says. One limitation is that analysis must await data download. "We can run an experiment that last several days only to find out at the end that the electrode was in the wrong place and we get nothing in terms of spikes," he says. A general limitation is battery size, but for aquatic animals the Styrofoam helps.

\section{Stop the noise}

With wireless systems, signal and signal processing are crucial. "The higher impedance the electrodes are, the more noise they generate," says Vecht. The signal from the electrodes must be greater than the noise from the electronics. Some labs choose electrodes made of non-traditional materials to reduce noise, and he points to the Neuropixels initiative devoted to designing and making silicon probes to record neural activity from many neurons. It's funded by Howard Hughes Medical Institute, Wellcome Trust, Allen Institute for Brain Science and Gatsby Charitable Foundation. Vecht likes the especially compact electronics from Intan Technologies, a company founded by Reid Harrison, who has been important in the Open Ephys movement, as has Jakob Voigts at MIT and others. Harrison left a faculty post at the University of Utah to found the company, which develops microchips to miniaturize the analog front end of a multichannel neural recording system.

"Our chips bridge the gap between electrodes and a digital data stream," says Harrison. Before these chips, every amplifier channel in an electrophysiology measurement system required dozens, if not hundreds, of electrical components such as low-noise operational amplifiers, resistors, capacitors and analog-to-digital converters, all of which made multichannel recording 
devices large and heavy. Starting in 2010, he and his team began developing microchips with onboard components for 16,32 or 64 low-noise amplifier channels with integrated analog-to-digital conversion. The 64-channel chip measures $7 \times 9$ millimeters, is lightweight and has low power needs.

What's tough about wireless neural recording, says Harrison, is that signals picked up by electrodes in the brain are extremely small and weak. A typical signal level is 100 microvolts, "and it takes sophisticated electronics to isolate and amplify these signals to sufficient strength that they can be digitized with high fidelity."

It's also challenging to build amplifiers that do not add much noise from the thermal motion of electrons in their transistors. And there is "a fundamental tradeoff between power and noise in amplifier design," he says. Most techniques for reducing the inherent noise in amplifiers make it possible to resolve tiny neural signals, but they increase power consumption. "This is a problem for wireless systems which must operate for hours or days from small batteries," he says. He developed circuit design techniques to optimize this tradeoff between power and noise "so that you can have the best of both worlds," he says. Intan's line of stimulation/ amplifier chips add electrical stimulation capability to the low-noise amplifiers so users can elicit neural activity with brief pulses of current. Some labs use the system for optogenetic stimulation.

Across experimental neuroscience, especially in wireless systems, Harrison sees "the desire to record activity from more and more neurons simultaneously," he says. It's hard mainly due to the limited bandwidth of wireless connections. Low-power wireless transmitters like Bluetooth can only handle perhaps a dozen channels of neural activity. Wi-Fi can handle more, but it's power hungry and reduces battery life considerably. One alternative is the approach Vecht takes: to store data locally on a microSD card. That can also mean, for some experiments, says Harrison, that one runs out of storage space. Some labs and companies build systems to transmit data and energy wirelessly.

\section{Wireless power}

To labs with electrical engineering challenges, Stanford University engineer Ada Poon renders what she calls a "Santa Claus" service. "Engineers are very versatile," she says. Some of her projects have involved optogenetic stimulation of neurons in the brain of a mouse or ways to influence the plasticity of fat cells in reaction to diet or exercise. "I feel it's more fun if in every

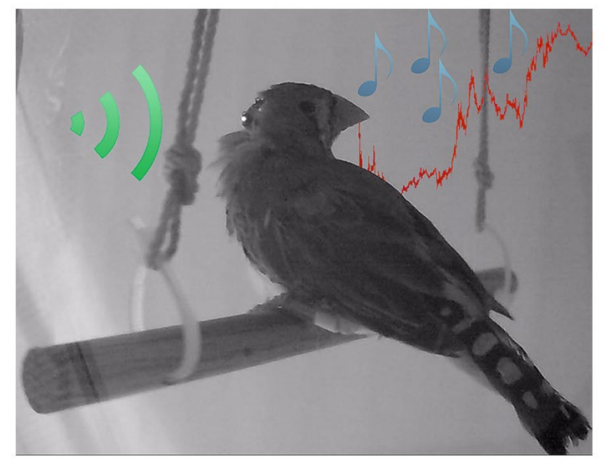

Poon's latest system is a one-square-millimeter silicon chip with an antenna that transmits data to an external storage device and has a low-power transceiver outside the cage. This device is designed to stay in the animal for a month and is encapsulated in parylene to reduce infection risk. In this experiment, focused on repairing stroke-damaged neurons, it tracks stem cell differentiation in the brains of mice. In some of Poon's projects, stimulation and recording are done at different locations in the brain. To resolve the data, each device has a number. "It's like having two different cell phone numbers," she says. The system can be used in an experiment with a number of socially interacting animals.

The lab of John Rogers at Northwestern University's Querrey Simpson Institute for Bioelectronics (QSIB) is all about pliable, stretchable materials and electronics for use in biological tissues. He and his team have invented numerous technologies for use in wireless neuroscience ${ }^{4,11}$. The lab's "base system" of implantable devices has been commercialized for research use in neuroscience, cardiology, transplantation medicine and microfluidic drug delivery, says Anthony Banks, who was in Rogers' lab at the University of Illinois Urbana-Champaign. Banks is director of QSIB's Engineering Research and is CEO of NeuroLux, which commercializes technology from the Rogers lab as well as other labs.

The devices from the Rogers lab fit on a fingertip and weigh around 30 milligrams. The suite of wireless devices include some powered by radio frequency and others by near-field wireless power such as the kind used in payment systems. The devices can be used to apply optogenetic stimulation of neurons in the brain, spinal cord or peripheral nervous system. They are bendable and equipped with electrodes, micro-LEDs and electronics.

Biomedical engineer Philipp Gutruf is a former postdoctoral fellow in the Rogers lab and his core expertise is wireless power transfer. Gutruf's devices build and expand on work he did in the Rogers lab, and he is now at the University of Arizona with appointments in neuroscience, biomedical engineering, and electrical and computer engineering. He is developing and using devices to optogenetically stimulate neurons in the brains of rodents and birds. "We can deliver light, we can record temperature, we can do many things," he says. A bat project is just starting up, he says.

The lab's wireless devices with circuits and electrodes use wireless power transfer in such a way that neuromodulation in birds 


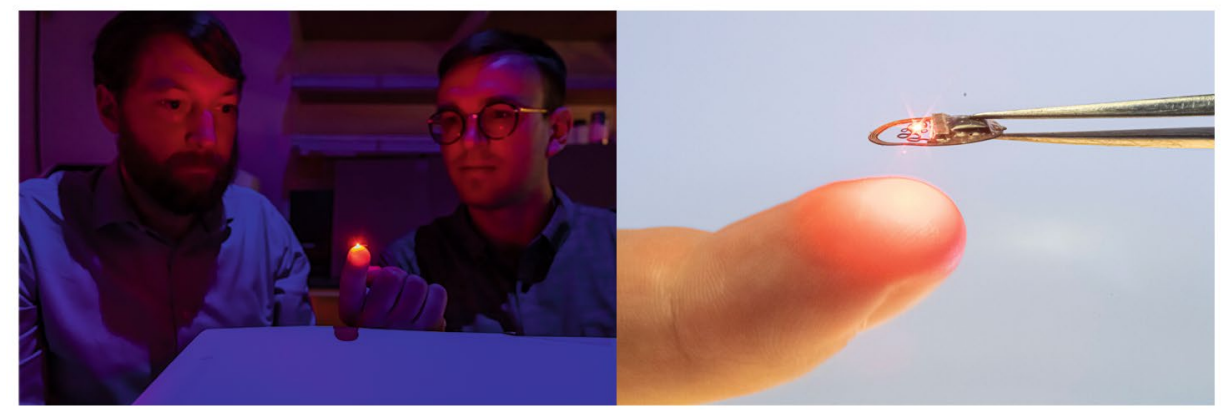

Philipp Gutruf (left), graduate student Jakubas Ausra and colleagues develop wirelessly powered devices for transcranial optogenetic stimulation. Credit: Gutruf lab, Univ. of Arizona

is possible, he says. He wanted to avoid the weight of a battery in a zebra finch project his team worked on with Julie Miller's team at University of Arizona, which studies zebra finches and speech and language more generally. The team tested the device with freely behaving birds in a cage around $35 \times 35 \times 20$ centimeters in size and optogenetically stimulated vocalization ${ }^{12}$. They measured temperature, which helps to capture behavior changes associated with sleep-wake cycles. "The technological challenge here is the volume that you can cover," says Gutruf. The device has electronic components atop copper layers that define the electrical circuit, he says. Some device versions have components on the top and bottom layer and some use only the top layer. The device itself is encapsulated in parylene.

Power is relayed to the device with near-field technology; this resonant magnetic power transfer at 13.56 megahertz is at a frequency not absorbed by the body. Given the widespread use of this technology, many commercial components are available, which makes device construction practical and affordable, he says. Energy is sent from the external source to the antenna on the subdermally implanted device. The team saw that they were able to cover the cage area evenly, wherever the birds chose to be. The current drives the electronics for measurement and stimulation modalities and powers the infrared transmission of data from the implant back to a data collection hub. A red LED placed at the back of the bird's head delivers data in the form of a blinking pattern back to the instrument hub. It's a digital modulation of the recorded signal. The scientists optogenetically modulated the bird's vocalization with stimulation of area X, a basal ganglia brain nucleus in male zebra finches involved in song. The birds had received, via viral vector, channelrhodopsin delivered to the ventral tegmental area, where it was taken up by dopaminergic neurons that project to area $\mathrm{X}$.

In wireless optogenetic experiments with mice ${ }^{13}$, the Gutruf team explored transcranial optogenetic stimulation with the device. "We can make very high intensity pulses of light that penetrate the skull and into the brain without actually drilling a hole in it," he says. In such experiments, light is pulsed. The researchers developed a way to digitally manage capacitive energy storage between light pulses, and this extends the length and range of experiments. Gutruf has projects ongoing that involve stimulating the spinal cord and the musculoskeletal system; in these projects the device is embedded in growing bone.

Practically, wireless systems always require parameter tweaking, says Gutruf. Every experiment and configuration has challenges, but researchers can approach him to help troubleshoot systems. "The devil's always in the details," he says. He is distributing his system through NeuroLux and plans to keep evolving it, get it into many labs and expand its uses. Since it does not need to be recharged, experiments can last weeks or months and there is no need to touch the animal, which can perturb behavior. In the space for wireless devices in neuroscience, Gutruf sees a growing demand for optogenetics applications. Optogenetics has a cell specificity that he likes for investigations not possible with electrical stimulation.

The weight reduction and speed of wireless measurement systems are remarkable, says Hiroki Koda, a neuroscientist at Kyoto University. Experiments are becoming easier to do, which means measurement technology needs to be expanded. Now or in the near future, the collection of big data could enable a system that can automatically analyze social behavior using artificial intelligence, especially in an unsupervised way. "We also need to develop mathematical theories to understand the synchronization of neural activity with other individuals," he says. And it would be useful to have a "simultaneous social group laboratory" that can control and monitor multiple individuals in a social group.

Says Grieves, wireless technology and 3D tracking are all improving at a remarkable pace. There are many advantages when untethered animals can move freely and express natural behaviors. Device developers have done well and, he says, "the onus is on researchers now to stop thinking two-dimensionally and start planning to make use of this technology."

Vivien Marx ${ }^{凶}$
Nature Methods.
$\rrbracket_{\text {e-mail: V.Marx@us.nature.com }}$

Published online: 27 September 2021 https://doi.org/10.1038/s41592-021-01281-6

References

1. Shin, G. et al. Neuron 93, 509-521.e3 (2017).

2. Gutruf, P. et al. Nat. Electron. https://doi.org/10.1038/s41928-0180175-0 (2018).

3. Won, S. M. et al. Nat. Biomed. Eng. https://doi.org/10.1038/ s41551-021-00683-3 (2021).

4. Yang, Y. et al. Nat. Neurosci. 24, 1035-1045 (2021).

5. Hafting, T. et al. Nature 436, 801-806 (2005).

6. Grieves, R. M. et al. Nat. Neurosci. https://doi.org/10.1038/ s41593-021-00907-4 (2021).

7. Ginosar, G. et al. Nature 596, 404-409 (2021).

8. Eliav, T. et al. Science 372, eabg4020 (2021).

9. Montgomery, K. L. et al. Nat. Methods 12, 969-974 (2015).

10. Kajima, K. et al. Nat. Commun. 11, 1730 (2020).

11. Park, S. I. Nat. Biotechnol. 33, 1280-1286 (2015).

12. Ausra, J. et al. Nat. Commun. 12, 1968 (2021).

13. Ausra, J. et al. Proc. Natl Acad. Sci. USA 118, e2025775118 (2021). 